\title{
Civilisations
}

Revue internationale d'anthropologie et de sciences

humaines

60-1|2011

Mobilisations et dynamiques identitaires en Amérique andine

\section{La participation de la Confédération Indigène de Bolivie}

à la vie politique nationale bolivienne (1982-2010)

Laurent Lacroix

\section{(2) OpenEdition}

\section{Journals}

Édition électronique

URL : http://journals.openedition.org/civilisations/2775

DOI : $10.4000 /$ civilisations. 2775

ISSN : 2032-0442

Éditeur

Institut de sociologie de l'Université Libre de Bruxelles

Édition imprimée

Date de publication : 31 décembre 2011

Pagination : 103-119

ISBN : 2-87263-035-X

ISSN : 0009-8140

\section{Référence électronique}

Laurent Lacroix, «La participation de la Confédération Indigène de Bolivie », Civilisations [En ligne],

60-1 | 2011, mis en ligne le 12 février 2015, consulté le 19 avril 2019. URL : http://

journals.openedition.org/civilisations/2775; DOI : 10.4000/civilisations.2775 


\title{
La participation de la Confédération Indigène de Bolivie
}

\author{
à la vie politique nationale bolivienne (1982-2010)'
}

\author{
Laurent LACROIX
}

\begin{abstract}
Résumé : Depuis son apparition dans les années 1980, la Confédération Indigène de Bolivie (CIDOB) se présente comme un acteur important dans la vie politique bolivienne. Par des actions spécifiques et des propositions novatrices, cette organisation représentative des peuples autochtones des Basses Terres est parvenue à hisser les questions de la diversité culturelle et de la territorialité ethnique dans les débats politiques. Son activisme a ainsi permis à ses affiliées de sortir d'un anonymat prolongé et d'interférer dans une histoire nationale jusqu'alors autocentrée sur la région andine. Des réformes néoindigénistes des années 1990 à la Constitution de 2009 qui prévoit l'instauration d'un État plurinational, la CIDOB a su négocier la considération de son projet politique. Cet article examine successivement l'émergence de cette organisation multiethnique non andine, ses revendications, ses modes d'action collective, ses conquêtes politiques et les nouvelles perspectives de mobilisation qui s'érigent pour les peuples autochtones de Bolivie avec la construction effective d'un État plurinational.
\end{abstract}

Mots-clés : Bolivie, Mouvement Indigène, CIDOB, droits autochtones, État plurinational.

\begin{abstract}
Since its foundation in the 1980s, the Indigenous Confederation of Bolivia (CIDOB) appears as an important actor in the Bolivian political arena. This lowlands indigenous peoples representative organization succeeded in integrating the questions of the cultural diversity and the ethnic territoriality in the political debates by specific actions and innovative propositions. This activism so allowed its members to go out of a prolonged anonymity and to interfere in a national history until then auto-centered on the andean region. From the neoindigenist reforms of $1990 \mathrm{~s}$ until the Constitution of 2009 which plans the institution of a plurinational State, the CIDOB knew how to negotiate the consideration of its political project. This paper examines successively the emergence of this not andean multiethnic organization, its demands, its collective actions modes, its political experiences and the new perspectives of mobilization which set up for the indigenous peoples of Bolivia with the effective construction of a plurinational State.
\end{abstract}

Keywords: Bolivia, Indigenous movement, CIDOB, indigenous rights, plurinational State.

1. Cet article synthétise une partie de thèse de doctorat en sociologie intitulée « Indigènes et Politique en Bolivie » (IHEAL, Université Sorbonne Nouvelle- Paris 3, 2005). Celle-ci a été complétée par une enquête de terrain réalisée en 2010 qui s'inscrit dans le cadre d'une recherche collaborative financée par le Conseil Européen de la Recherche (ERC 249236) - SOGIP : « Scales of Governance : the UN, the State and Indigenous Peoples : self-determination at the time of globalization » (www.sogip.ehess.fr). 
Nous ne demandons pas ce qui existe. Nous demandons ce qui n'existe pas pour que cela existe.

C'est cela le rôle des peuples indigènes. José Bailaba Parapaino, Chiquitano, octobre 2010

$\mathrm{P}$ qui les menacent, de nombreux peuples autochtones recourent à l'utilisation politique de l'ethnicité (Morin et Saladin d'Anglure 1995). En Amérique latine, cette stratégie consiste à politiser l'ethnicité, c'est-à-dire à faire " reconnaître la dimension ethnique comme une des dimensions légitimes d'appartenance et de relation dans le champ politique » (Gosselin 2001 : 126), sans toutefois aller jusqu'à une ethnicisation de la vie politique qui se traduirait par l'instauration officielle de frontières ethniques dans le champ des relations politiques et partisanes entre certaines catégories de groupes sociaux. Si quelques discours ethnonationalistes, minoritaires et conjoncturels, peuvent émerger ponctuellement et localement dans la région andine, notamment en Bolivie (Lacroix 2005 ; Lavaud 2006), l'ethnicisation croissante des identités collectives et des demandes sociales n'implique pas pour autant systématiquement, loin de là, un mouvement de repli communautaire ou l'institutionnalisation d'ethnicismes en Amérique latine remettant en cause l'existence ou l'intégrité des États (Gros 2007). Cette caractéristique invite à être prudent dans l'analyse des transformations en cours et à établir, par exemple, une différence entre les processus d'ethnogenèse, d'ethnification et d'ethnicisation émergeant dans le cadre des politiques multiculturalistes mises en place en Amérique latine (Boccara 2010).

Qu'ils apparaissent comme indiens, indigènes, autochtones ou originaires selon les régions et les périodes historiques, les peuples autochtones d'Amérique latine, majoritairement pacifiques, proposent une (re)définition des sociétés nationales et des États (Le Bot 1994) en revendiquant l'instauration d'un État plurinational qui permet d'envisager un dépassement du modèle historique « uninational » des nations latino-américaines hérité de la colonisation (Acosta et Martínez 2009). En Bolivie et en Équateur, où l'instauration d'un État plurinational est prévue par la Constitution, il s'agit d'aller au-delà de la reconnaissance de droits collectifs promue par les politiques multiculturalistes libérales des années 1990 pour construire, de manière structurelle, « l'unité [nationale] dans la diversité »² (Lacroix 2011).

La Confédération Indigène de Bolivie (CIDOB) a largement contribué à ce changement notable de paradigme politique en mobilisant l'ethnicité comme ressort de revendication, de mobilisation et d'action collective. L'analyse des stratégies adoptées par cette organisation interethnique depuis sa fondation en 1982 montre combien l'identité ethnique a une valeur performative dans le champ politique bolivien « en ce sens qu'elle finit effectivement par orienter le comportement des acteurs sociaux et par leur offrir un horizon de sens et une possibilité de mobilisation » (Gallisot, Kilani et Rivera 2000 : 109).

2. Principal slogan des organisations autochtones revendiquant l'instauration d'un État plurinational en Amérique latine. 
Le cas de la CIDOB met en évidence le caractère à la fois interactionnel, situationnel, dynamique et flexible de l'ethnicité (Barth 1969), et renvoie dos-à-dos deux courants de pensée en vogue et en forte concurrence en Amérique latine face à la participation politique croissante des peuples autochtones. D'un côté, l'indianisme s'attelle en effet à valoriser des perceptions et des pratiques qui seraient propres aux populations d'origine amérindienne (qui, bien souvent, se révèlent être reconstruites pour l'occasion) et à questionner la légitimité historique de la société nationale. Ce courant, plutôt andin (ou andinocentrique), est développé depuis les années 1970 par quelques intellectuels éloignés du mouvement autochtone et de ses revendications. À l'opposé, le constructivisme radical tend pour sa part à considérer l'ethnicité comme un paradigme identificatoire artificiel auquel contribuerait grandement la communauté internationale et qui menacerait les unités nationales latino-américaines. Alors qu'il met brillamment en lumière les évolutions historiques des catégories identitaires, ce courant déconsidère le rôle et les propositions politiques des peuples autochtones, pris dans sa croisade quasi-obsessionnelle contre toute forme d'essentialisme. En s'imposant progressivement comme un acteur important de la scène revendicative et politique nationale en Bolivie, la CIDOB permet d'appréhender, comme cela est en partie suggéré par l'approche barthienne, les mobilisations ethniques des peuples autochtones en termes de stratégies politiques et au-delà, l'importance de l'ethnicité dans le champ politique en Amérique latine, notamment en Bolivie.

La CIDOB constitue depuis plusieurs années un acteur important et influent dans la vie politique bolivienne. Elle présente des projets de réforme innovants prônant la redéfinition d'une nation multiculturelle et l'instauration d'un État plurinational tout en menant des mobilisations d'envergure qui permettent aux peuples autochtones des Andes et des Basses Terres d'acquérir de nouveaux droits dans un contexte international propice à la question autochtone. En ce sens, l'expérience de la CIDOB constitue une composante essentielle dans l'histoire du mouvement autochtone en Amérique latine.

\section{La formation de la CIDOB}

\section{Histoire d'une alliance identitaire et stratégique}

\section{L'émergence du mouvement autochtone dans les Basses Terres}

Depuis la colonisation, les populations autochtones des Basses Terres de Bolivie n'ont cessé d'être placées sous tutelle. D'abord religieuse, avec l'implantation de missions jésuites et franciscaines entre 1681 et 1767 qui ont constitué des lieux d'ethnogenèses issues de cohabitations forcées entre les groupes précolombiens locaux; puis laïque, de 1768 à 1953, sous l'autorité d'administrateurs locaux ou de patrons d'haciendas dont la principale activité était de tirer profit de la main d'œuvre colonisée pour exploiter les terres qu'ils avaient spoliées (Lacroix 2005).

La réforme agraire de 1953, qui fait suite à la révolution nationale de 1952, ne change guère cette situation générale. La mise sous tutelle se poursuit. Les droits agraires des groupes sylvicoles ou communautés natives sont placés sous la responsabilité de l'État, des évêchés ou des grands propriétaires locaux. L'Institut Linguistique d'Été, une institution baptiste chargée par l'État bolivien d'assurer l'éducation bilingue des populations autochtones de cette région, perpétue l'entreprise d'évangélisation et d'acculturation initiée par les missionnaires jésuites et franciscains (Castro Mantilla 1997). Durant trois décennies, l'État bolivien ne reconnaît 
pas de droits territoriaux aux groupes ethniques marginaux des Basses Terres. Les aires d'habitat des populations autochtones de cette région se réduisent progressivement avec l'installation massive de paysans andins (projets dits de colonisation) et la mise en place de politiques développementistes favorisant l'agro-industrie et le système de grande propriété.

À la fin des années 1970, des représentants des peuples Ayoréodes, Guarayo, Guaraniizoceño, Chiquitano, Ava-Guarani et Weenhayek se rencontrent à plusieurs reprises pour discuter de la situation commune aux populations autochtones des Basses Terres. Dans les archives de la CIDOB, cette situation est décrite en termes de spoliation agraire historique et de marginalisation tant économique que politique. Les réunions peuvent se réaliser grâce à l'aide financière d'un petit groupe d'anthropologues allemands travaillant sur place depuis quelques années et dont il est difficile d'évaluer précisément l'influence dans le processus de fabrique de l'ethnicité alors en cours dans cette région. Il est toutefois fort probable que ces derniers aient influencé la constitution de la future confédération pan-ethnique même si les dirigeants autochtones avaient, chacun de leur côté, des projets de cet acabit. Ces derniers n'ayant pas les moyens de réaliser les rencontres qu'ils souhaitaient, ils ont sollicité des anthropologues avec qui ils collaboraient depuis plusieurs années.

En 1982, la Centrale Indigène ${ }^{3}$ de l'Orient Bolivien est fondée pour faire face aux grands projets de développement économique et d'aménagement planifiés pour la région, projets qui menacent les aires d'habitat des populations autochtones.

\section{Les composantes de l'ethnicité}

Le caractère ethnique adopté par la CIDOB repose sur un double processus de différenciation. Le premier se réalise à l'égard des petits agriculteurs andins installés dans les Basses Terres à l'issue des vagues de «colonisation » planifiées et organisées par l'État (Loi de Colonisation 1966) puis libres. Il s'agit pour la CIDOB de proposer une alternative au syndicalisme paysan prédominant en milieu rural depuis les années 1950, incarné par la Confédération Syndicale Unitaire des Travailleurs Paysans de Bolivie (CSUTCB), dont les revendications portent essentiellement sur l'attribution de parcelles individuelles de terre mais ignorent la question des communautés rurales dans les Basses Terres. Les populations affiliées à la CIDOB cherchent, pour leur part, à établir une organisation plus autonome des partis politiques, moins verticale que le syndicalisme et surtout, recherchent le moyen d'obtenir des terres collectives.

Ce dernier objectif constitue la base du second processus de différenciation effectué par la CIDOB. Cette fois-ci, c'est à l'égard des élites politiques et économiques locales, d'origine étrangère (descendants de colons ou de nouvelles vagues de migrations internationales), avec lesquelles les populations indigènes sont en compétition pour l'accès aux ressources naturelles. Cette élite contrôle le marché des terres et contribue à l'avancée du front agricole menaçant les espaces d'habitat des populations indigènes. Ainsi, la CIDOB vient combler un vide dans l'espace des organisations représentatives dans la région des Basses Terres et vient bousculer l'idée d'une indianité uniquement andine dans l'imaginaire politique national.

3. Terme hispanophone équivalent à celui d'autochtone. Dès lors, le terme « indigène » sera utilisé en respect de la terminologie locale. Le terme « autochtone » sera utilisé en référence à la catégorie internationale ou de manière générique pour évoquer l'ensemble des peuples autochtones de Bolivie, des Basses Terres et des Andes. 
Lors de son $6^{\mathrm{e}}$ Congrès en 1987, la CIDOB parvient à déterminer son principal objectif qui consiste en la « défense des droits fondamentaux des peuples indigènes du pays ». Une première plate-forme revendicative commune est établie, insistant sur la reconnaissance des territoires et des communautés indigènes, l'autonomie des peuples, la consolidation d'une organisation nationale et la mise en place de programmes d'éducation bilingue et biculturelle (Lacroix 2005). Peu à peu, la représentativité de la CIDOB s'étend à un niveau national. Entre 1987 et 1997, sept organisations départementales et près de quatre-vingts organisations régionales et locales sont fondées et rejoignent les rangs de la CIDOB. Au milieu des années 1990, plus de mille communautés autochtones y sont affiliées. La Centrale Indigène de l'Orient Bolivien se transforme en Confédération Indigène de l'Orient, du Chaco et de l'Amazonie de Bolivie, puis quelques années plus tard en Confédération des Peuples Indigènes de Bolivie. En mars 1990, elle est officiellement reconnue comme entité représentative des peuples autochtones des Basses Terres de Bolivie par l'État bolivien. Ce changement de statut s'accompagne d'une autonomisation organisationnelle et financière concertée vis-à-vis des anthropologues ayant favorisé sa fondation. En 2010, la CIDOB représente trente-quatre peuples indigènes.

La croissance de cette organisation ne résulte pas d'un processus endogène. Le contexte international a joué un rôle important dans le développement et la consolidation de la CIDOB. Celle-ci apparaît dans le paysage politique bolivien au moment où l'anthropologie vit un bouleversement des idées et des approches. À ce moment en effet, les peuples autochtones commencent à être considérés comme des acteurs sociaux et politiques à part entière par des anthropologues américanistes (Morin et Saladin d'Anglure 1995). Ces derniers, inspirés par les deux déclarations des Barbades (1971 et 1977) et la nouvelle éthique interventionniste proposée par plusieurs organisations non gouvernementales, notamment scandinaves, s'engagent activement dans la défense des peuples autochtones et la promotion de leurs droits.

Dès sa fondation, la CIDOB mobilise l'ethnicité pour le contrôle d'espaces politiques et économiques, propres et autonomes. La stratégie de politisation de la différence culturelle émane d'une longue réflexion interne soutenue par des anthropologues engagés mais également au fil des rencontres avec d'autres organisations autochtones du continent, en particulier celles de la région amazonienne avec lesquelles la CIDOB fonde, en 1983, la Coordination des Organisations Indigènes du Bassin Amazonien (COICA).

La CIDOB n'a jamais proposé une définition claire de son caractère indigène. Comme cela a déjà été mentionné, l'indigène bolivien se distingue du paysan andin et des groupes dominants locaux d'origine étrangère. Voilà pour l'aspect local de la définition. Toutefois, les archives de l'organisation, notamment les procès-verbaux des congrès, offrent quelques éléments complémentaires quant au processus de construction identitaire dans une perspective plus globale. Au fil des discussions internes, les indigènes de la CIDOB s'avèrent être des paysans pauvres, aux us et coutumes propres non reconnus par l'État bolivien qui les ignore et se désintéresse de leur situation, et dont les aires d'habitat sont affectées par l'avancée permanente d'acteurs économiques externes. Ces précisions font écho aux discussions autour des standards de l'autochtonie qui se mettent en place simultanément au niveau international et qui s'orientent vers une première définition établie sur quatre critères faisant aujourd'hui consensus dans ces arènes : les peuples autochtones sont des groupes préexistant aux nations 
modernes (origine précoloniale), culturellement minoritaires, politiquement dominés et s'auto-définissant comme « autochtones » (Saugestad 2001).

De la même manière, un glissement conceptuel et terminologique de Confédération Indigène à Confédération des peuples indigènes de Bolivie s'opère alors que l'expression de peuples autochtones s'impose au sein des agences internationales (Bellier 2007 et 2009). Cette nouvelle catégorie officielle ne relève pas seulement du droit international mais transcende les dimensions politiques et identitaires lorsqu'elles sont mobilisées par les peuples autochtones pour interpeller les États (Gagné et Salaün 2009). L'utilisation du terme peuples autochtones implique un lien direct entre ethnicité et politique (Bellier 2009).

Alors qu'elle ne participe pas ou peu aux instances internationales attentives à la question autochtone, la CIDOB parvient toutefois à suivre les évolutions des débats sur l'autochtonie au niveau international et utilise tous les ressorts possibles pour affiner, avec un maximum d'efficacité politique, son utilisation stratégique de l'ethnicité. Le contact avec la sphère internationale se réalise de manière indirecte, par les rencontres avec d'autres organisations autochtones du continent mais aussi et surtout par le biais des organisations non gouvernementales ${ }^{4}$ ou de services de coopération (Pays-Bas, Danemark) qui jouent un rôle d'intermédiaire aussi important que le soutien technique, humain, stratégique et financier qu'elles apportent à la CIDOB depuis sa fondation. La Confédération indigène compte très peu d'entrepreneurs ethniques (Morin et Saladin d'Anglure 1995) qui se rendent dans les instances internationales pour présenter la situation des peuples indigènes des Basses Terres de Bolivie. Rares sont les représentants autochtones de cette région qui assument des fonctions à l'étranger ou ont une expérience internationale même si leur nombre croît de manière régulière. Cette caractéristique ne paraît pas représenter un obstacle pour la CIDOB dont les revendications sont explicites et l'action efficace dans le champ politique bolivien.

\section{Le territoire comme première revendication}

À l'instar de nombreuses organisations autochtones, la CIDOB revendique le droit à la différence culturelle, à l'autodétermination et à l'autonomie pour les populations indigènes des Basses Terres de Bolivie. Cette triple revendication se traduit par une série de demandes dont la formulation et la priorité varient selon la conjoncture sociale et politique du pays, les législations en vigueur et la capacité de mobilisation de la direction de la CIDOB. Toutefois, les objectifs de fond demeurent. Les peuples indigènes des Basses Terres de Bolivie demandent à être constitutionnellement reconnus et légalement considérés ainsi que leurs territoires, leurs langues, leurs us et coutumes, leurs droits coutumiers, leurs systèmes internes de gouvernement et d'administration locale, leurs médecines traditionnelles. Ils réclament un certain nombre de droits spécifiques comme celui d'obtenir des titres de propriété collective sur des espaces occupés traditionnellement ; celui d'accéder prioritairement et de gérer les ressources naturelles présentes dans ces espaces ; celui de bénéficier d'une éducation bilingue et pluriculturelle qu'ils estiment fondamental pour la « survie » de leur culture ; ou encore celui de disposer d'un pluralisme juridique au niveau local.

4. Entre autres, International Work Group for Indigenous Affairs (IWGIA), Oxford Commitee for Famine Relief (OXFAM), Apoyo para el Campesinado del Oriente Boliviano (APCOB), Centro de Estudios Jurídicos y Sociales (CEJIS). 
Toutes ces revendications sont importantes et interdépendantes pour les organisations indigènes des Basses Terres de Bolivie. Toutefois, le thème de la territorialité se détache de tous les autres (Lacroix 2005). Pour la CIDOB, l'existence d'un « territoire indigène " implique une unité spatiale intégrale dans laquelle les populations autochtones contrôleraient l'ensemble des ressources naturelles, exerceraient leur autonomie politico-administrative et assumeraient leurs histoires et leurs cultures. Le territoire constitue un point de départ fondamental pour traiter les autres problèmes auxquels sont confrontées les communautés indigènes de Bolivie (Lema 1997).

Dans le discours de l'organisation interethnique, la notion de territoire apparait centrale et intimement liée à celle des peuples indigènes. Cette relation constitue le ciment entre les différentes composantes de la CIDOB. Elle se présente comme le principal élément fédérateur. Dès 1988, la CIDOB propose l'instauration d'espaces territoriaux protégés au bénéfice des populations indigènes qui en font la demande. Cette revendication, confortée l'année suivante par la convention 169 de l'OIT relative aux peuples autochtones et tribaux, attire quelques organisations conservationnistes nationales et transnationales qui perçoivent les populations indigènes comme protectrices de l'environnement et proposent à la CIDOB leurs conseils juridiques et stratégiques (Liberman Cruz et Godínez Gutiérrez 1992 ; Cejis 1992).

Si la notion de territoire reste très variable selon les groupes ethniques (Chirif, Garcia, Chase Smith 1991), la CIDOB parvient, au fil de ses congrès et au contact des organisations internationales et de l'État bolivien, à élaborer une définition claire et consensuelle mentionnée dans son Projet de Loi Indigène (1992). «Les territoires indigènes sont les terres traditionnellement occupées ou possédées par les peuples indigènes (...) qui constituent leur habitat naturel et l'espace socio-économique utilisé pour leurs activités de production, de chasse, de pêche, de cueillette, d'agriculture, d'élevage (...) ainsi que les aires imprescriptibles nécessaires pour garantir leur croissance démographique et leur développement durable » (art. 9). Ces territoires doivent être inaliénables et indivisibles (art. 10). L'État doit garantir la propriété collective de ces territoires aux populations bénéficiaires et leur accorder le droit d'administrer et de gérer les ressources naturelles (art. 11 et 19). Les peuples indigènes devront être consultés avant toute autorisation d'exploitation des ressources souterraines sur leurs territoires (art. 12).

La notion de territoire autochtone acquiert une nouvelle dimension au fil des troubles sociaux qui se succèdent entre 2000 et 2005 et qui aboutissent à l'instauration d'une Assemblée Constituante entre 2006 et 2008. Dans ce cadre, de nombreux projets de " rénovation nationale » émanant de divers secteurs de la société, dont la CIDOB, ont considéré la question territoriale autochtone dans la perspective d'une réorganisation politico-administrative de l'État bolivien. Celle-ci apparaît sous le label générique des « autonomies indigènes » qui sera décrit ultérieurement dans ce texte et qui fait écho aux autonomies départementales revendiquées par les mouvements régionalistes des Basses Terres (Lacroix 2006). 


\section{Marches revendicatives et propositions législatives comme modes d'action collective}

Ce que de nombreux observateurs appellent le « réveil indien » ou la « réindianisation » en Amérique latine ${ }^{5}$ correspond à l'émergence, depuis les années 1970, de mouvements ethniques autochtones qui adoptent des formes innovantes de participation politique caractéristiques des nouveaux mouvements sociaux (García Serrano 2001). Pour les mouvements ethniques autochtones, celles-ci se traduisent, entre autres, par une autonomie vis-à-vis des partis politiques ou de l'État, une organisation interne plus horizontale et moins centralisée que celle des syndicats et favorisant la prise de décision collective, des revendications principalement identitaires et culturelles impliquant des changements structurels comme l'instauration d'un État plurinational et des modes d'actions peu institutionnalisés comme des marches ou des occupations d'espaces publics et médiatiques.

Pour présenter ses demandes à l'État bolivien, la CIDOB alterne entre la présentation de projets politiques ou de propositions législatives et l'organisation de marches revendicatives. Ce double mode opératoire résulte de l'adoption d'une stratégie d'action collective particulière dite constitutionnaliste et légaliste définie en 1989 lors $8^{\mathrm{e}}$ congrès national. Celle-ci consiste d'une part à saisir toutes les opportunités offertes par les nouvelles lois afin de faire valoir les droits revendiqués par ses affiliées et d'autre part, à élaborer des propositions de réforme constitutionnelle ou législative. Les formes d'action et les revendications doivent respecter le champ de la légalité et le dialogue permanent avec l'État devient une condition recherchée.

Depuis le début des années 1990, la CIDOB est omniprésente sur la scène politique bolivienne et obtient des résultats conséquents. L'activisme de la confédération indigène est tel que « le protagonisme dominant dans les mouvements indigènes s'est déplacé géographiquement des Andes à l'Orient » (Calla Ortega etMolina Barrientos 2000 : 27). Pour mieux comprendre l'impact de la CIDOB dans la vie politique bolivienne, plusieurs actions primordiales doivent être mentionnées. Celles-ci permettront de constater quelques exemples d'utilisation politique de l'ethnicité et de mesurer ses conséquences en Bolivie.

\section{La contribution à l'adoption du multiculturalisme}

Au mois d'août 1990, la CIDOB organise une marche «Pour le Territoire et la Dignité » pour protester contre la réduction des aires d'habitat des peuples indigènes des Basses Terres et pour revendiquer des territoires permettant de garantir celles-ci. Cette action marque la volonté de ces peuples à devenir des composantes actives d'une « nation plurielle et démocratique » (Libermann Cruz et Godínez Gutiérrez 1992). Le projet d'instaurer un État plurinational, proposé initialement par le katarisme ${ }^{6}$, un syndicalisme de type indianiste actif dans les années 1970, réapparaît au sein du mouvement indigène (Albó 1991).

La mobilisation provoque un électrochoc au sein de la société nationale qui découvre l'amplitude de la diversité culturelle du pays (Lehm 1999). Elle est soutenue par une large frange de la population et inspire les organisations autochtones andines en rupture avec le

5. Parmi les nombreux ouvrages sur la question, citons De Certeau et Materne 1977, Labrousse 1984 et plus récemment, Le Bot 2009.

6. De Tupac Katari qui fut le meneur d'une rébellion indienne sur l'Altiplano bolivien en 1781. Sur le mouvement katariste, voir entre autres : Lavaud 1982, Pacheco 1992, Le Bot 1994. 
syndicalisme paysan prédominant qui créent, en 1997, le Conseil National des Ayllus et Markas du Qullasuyu (CONAMAQ). L'apparition de cette nouvelle organisation autochtone s'accompagne, comme pour la CIDOB dans les Basses Terres, d'un processus d'autoidentification et de différenciation. Depuis la fin des années 1990, les peuples autochtones andins se définissent comme nations originaires, se distinguant des peuples indigènes des Basses Terres et des paysans organisés en syndicats.

À l'issue de la marche, l'État bolivien reconnaît quatre territoires indigènes en 1990 puis cinq autres en 1991. En 1992, le gouvernement du président Paz Zamora ratifie la Convention 169 de l'OIT et relance le projet de Fonds de Développement des Peuples Indigènes d'Amérique latine et des Caraïbes. Il officialise l'éducation interculturelle bilingue puis, il adopte une loi sur l'Environnement qui reconnaît aux populations indigènes le droit à la participation, l'administration et l'exploitation des ressources naturelles dans les aires protégées. Enfin, il autorise la création d'une garde forestière indigène sur les territoires reconnus en 1990 (Marinissen 1995). Si tous ces acquis sont conquis dans une conjoncture nationale et internationale ${ }^{7}$ propice à la question des droits des peuples autochtones, la mobilisation de la CIDOB a joué un rôle fondamental dans l'obtention de ces résolutions. L'utilisation politique de l'ethnicité a, ici, largement dépassé les espoirs de la confédération indigène.

Forte du succès de sa marche, la CIDOB obtient de l'État bolivien, en plus des acquis mentionnés, la considération de sa proposition de Loi Indigène, une synthèse des revendications communes à toutes ses organisations affiliées. Une commission nationale est chargée de préparer une Loi des Peuples Indigènes. Des dissensions terminologiques et conceptuelles apparaissent rapidement autour des notions de « peuples », de « territoire » et de « droit territorial ». La CIDOB conteste la désubstantialisation de son projet politique. L'État bolivien se refuse à reconnaître des peuples autochtones craignant pour l'unité du pays. Comme cela se produit à l'échelle internationale, « l'enjeu essentiel de cette bataille conceptuelle n'est pas tant l'identification de l'autochtonie que le déni de la reconnaissance comme peuple » (Bellier 2007 : 129). La même frénésie unitariste rejette du même coup toute la dimension politique de la revendication territoriale de la CIDOB qui impliquerait une réorganisation complète de la division administrative de l'espace national et ne retient finalement qu'une dimension spatiale, les aires d'habitat, que l'État s'engagerait à protéger.

Malgré l'échec, la CIDOB marque le débat politique en bousculant les paradigmes et les conceptions de l'État et de la nation. Le gouvernement nouvellement élu du président Sánchez de Lozada promet de considérer les propositions politiques de la CIDOB tout en conservant l'orientation néolibérale annoncée des réformes économiques et politiques prévues (SNPP 1997). Le pouvoir exécutif engage une vaste refonte du cadre législatif national. Les changements sont conséquents pour les populations autochtones. La réforme constitutionnelle de 1994 définit la Bolivie comme une république unitaire, multiethnique et pluriculturelle (art. 1), et reconnaît les peuples autochtones (sans toutefois définir qui ils

7. Au niveau international, le début des années 1990 est marqué par des mesures importantes comme la Convention 169 de l'OIT (1989), la commémoration de la Découverte des Amériques (1992), la déclaration de l'année internationale des peuples autochtones et la proclamation d'une décennie internationale des peuples autochtones du monde par l'ONU (1993). 
sont), leurs droits et leurs " aires communautaires d'origine » (art. 171) ${ }^{8}$. Elle leur offre la possibilité d'une assistance juridique (art. 116) et élimine la nécessité de savoir lire et écrire pour être éligible (art. 221), ce qui facilite la participation électorale de nombreuses organisations ethniques. Ces changements constitutionnels amorcent une nouvelle ère, celle du multiculturalisme libéral ${ }^{9}$ qui se traduit par la mise en place de politiques néo-indigénistes. Celles-ci incluent des droits spécifiques pour les populations autochtones et s'insèrent dans un vaste mouvement de décentralisation participative lié à l'application de recettes néolibérales promues par les organismes internationaux (Gros 1999).

En 1994, la loi de Participation Populaire engage la municipalisation de tout le territoire national sur laquelle repose un système de démocratie participative locale. Pour la première fois depuis l'instauration de la République en 1825, les communautés autochtones sont reconnues juridiquement et constituent, au même titre que les communautés paysannes et les associations de quartiers urbains, les organisations territoriales de base (OTB) de la décentralisation participative qui est mise en place. La municipalisation a eu pour principal effet de générer une démocratisation de la vie politique locale. L'instauration du suffrage universel et de la participation populaire dans les municipalités a ouvert de nouveaux espaces politiques et citoyens dans lesquels se sont engagés de nombreux représentant(e)s autochtones et dont la présence s'est accrue tant dans les conseils municipaux que dans les " comités de vigilance ", organes chargés de contrôler l'exécution du plan participatif quinquennal de développement municipal (Lacroix 2005). Ce mouvement se traduit par une participation politique et citoyenne active des peuples autochtones au niveau local. Leur présence s'étend aux instances nationales, au Parlement, dans les ministères et les ambassades (Lacroix 2007). En 2005, l'élection d'Evo Morales à la présidence de la République marque le paroxysme d'une présence autochtone grandissante dans les sphères de pouvoir en Bolivie depuis l'adoption du multiculturalisme au début des années 1990.

\section{De la revendication territoriale à celle de l'autonomie indigène}

Aussi important soit l'impact de la décentralisation participative pour les populations indigènes en termes de citoyenneté, de participation et de représentation politique, la réorganisation politico-administrative du pays, qui est exclusivement fondée sur la municipalisation du territoire national, ne considère nullement la notion de territorialité ethnique revendiquée par la CIDOB. En conséquence, la confédération indigène entreprend une seconde marche « pour le territoire, les droits de participation politique et le développement » en 1996 alors qu'une nouvelle loi agraire, discutée depuis $1992^{10}$, doit être promulguée.

8. Article 171 de la Constitution politique réformée en 1994 : « Sont reconnus, respectés et protégés dans le champ de la loi, les droits sociaux, économiques et culturels des peuples indigènes qui résident sur le territoire national et plus spécialement ceux qui sont relatifs à leurs terres communautaires d'origine, garantissant l'usage et le profit des ressources naturelles, de leurs identités, leurs valeurs, leurs langues, leurs coutumes et leurs institutions ».

9. Pour une définition du multiculturalisme libéral, voir, entre autres : Taylor 1994, Tully 1999, Kymlicka 2001.

10. Depuis le début des années 1990, la CIDOB, la CSUTCB, la Chambre Agricole de l'Orient (CAO) et plusieurs organisations non gouvernementales ont multiplié les propositions engageant un débat national auquel le gouvernement a finalement dû participer en élaborant un projet de loi qui n'était pas prévu dans son programme politique en dépit d'une situation agraire conflictuelle. 
La CIDOB exige la titularisation immédiate des territoires reconnus par décrets suprêmes à l'issue de la marche de 1990 et la définition précise d'un processus de dotation territoriale dans la prochaine loi agraire. Des droits sociaux et politiques sont également revendiqués comme l'incorporation des travailleurs agricoles à la Loi Générale du Travail, la gratuité de l'obtention d'une pièce d'identité, la possibilité d'instaurer des municipalités indigènes dans le champ de la décentralisation participative et le droit de présenter des candidat(e)s aux élections sans l'intermédiation obligatoire des partis politiques (CIDOB 1996).

La loi de l'Institut National de Réforme Agraire (INRA) est promulguée en octobre 1996 et met fin à la marche de la CIDOB dont l'objectif principal est atteint. La nouvelle législation accorde le droit aux populations indigènes de bénéficier de Terres Communautaires d'Origine (TCO) mentionnées dans l'article 171 de la Constitution réformée en 1994 et en respect de la convention 169 de l'OIT. Celles-ci sont définies comme des « espaces géographiques qui constituent l'habitat des peuples et des communautés indigènes et originaires auxquels ces derniers ont traditionnellement eu accès et où ils maintiennent et développent leurs propres formes d'organisation économique, sociale et culturelle, de sorte qu'ils y assurent leur survie et leur développement. Elles sont inaliénables, indivisibles, irréversibles, collectives, composées par des communautés ou associations de communautés, insaisissables et imprescriptibles » (art. 41.5). Dix-sept articles précisent le statut de ces TCO, le processus de leur dotation et prévoient la participation des organisations autochtones aux commissions agraires départementales et nationale.

La loi INRA a des effets immédiats. Une vague de demandes de Terres Communautaires d'Origine surgit dans tout le pays. La CIDOB et ses affiliées ouvrent la voie, imitées par les organisations autochtones andines. Un vaste mouvement de territorialisation ethnique se produit en Bolivie. En 2007, plus de cent cinquante TCO sont déjà octroyées et couvrent près de $36 \%$ du territoire national selon l'État. De nouvelles demandes territoriales ont été déposées et cette superficie pourrait atteindre 42\% (Lacroix et Le Gouill 2010).

Malgré la forte demande de TCO, la territorialité indigène reste précaire. La formule des TCO proposée par la loi agraire ne répond pas à la revendication territoriale des peuples autochtones. Ces derniers ne bénéficient pas, comme le réclame la CIDOB, de droits exclusifs ou prioritaires sur ces espaces collectifs ni même de ressources décentralisées pour les administrer. Cette situation d'entre-deux juridique et politique favorise une superposition de droits qui génère de nombreux conflits sociaux entre les populations titulaires de TCO et divers concessionnaires (entreprises d'extraction, éleveurs, paysans) installés légalement ou illégalement dans les aires octroyées aux peuples autochtones (De Vries 1998 ; CIDOB 2000).

Maintenant ses objectifs en termes de dotation territoriale au bénéfice des peuples indigènes, la CIDOB imagine et développe le concept d'autonomies indigènes qui consiste à transformer les TCO ou les municipalités à majorité autochtone en circonscriptions ethniques intégrées à l'organisation politico-administrative de l'État. La proposition d'autonomies indigènes lie territorialité ethnique et autonomie juridictionnelle. Pour la CIDOB, les territoires ou municipalités autochtones doivent être dotés de gouvernements propres, autonomes, reconnus par l'État et assumer des compétences similaires à celles des autres entités territoriales subnationales (CIDOB 2008). En somme, il s'agit d'institutionnaliser des circonscriptions ethniques pleinement intégrées à l'organisation politico-administrative du pays qui permettraient aux peuples autochtones d'assumer une autonomie politique, 
législative et normative, exécutive, administrative, patrimoniale, territoriale et juridictionnelle en accord avec le champ juridique de l'État.

Pour mettre en place ces autonomies indigènes, une nouvelle constitution politique s'avère nécessaire. C'est dans cette perspective que la CIDOB se mobilise durant une décennie, aux côtés d'autres secteurs sociaux, pour l'instauration d'une assemblée constituante. Le pays traverse alors un cycle d'instabilité sociale et politique qui dure cinq années, de 2000 à 2005. Pendant cette période particulièrement agitée, les peuples indigènes organisent cinq marches $(2000,2002,2004,2006,2007)$ pour demander la convocation de l'assemblée constituante et l'institutionnalisation de territoires autochtones.

La mobilisation permanente de la CIDOB va générer des tensions internes liées à des divergences quant aux alliances stratégiques et politiques. D'un côté, des organisations indigènes départementales participent à des alliances intersectorielles nationales proches du Movimiento al Socialismo (MAS) d'Evo Morales qui incarne pour beaucoup de Boliviens le changement politique (Lacroix 2006). De l'autre, la direction nationale de la CIDOB tente de maintenir des relations avec les partis traditionnels au pouvoir et de poursuivre les réformes dans le cadre du multiculturalisme libéral. Cette distanciation relative aux différences stratégiques révèle l'émergence d'une nouvelle génération de dirigeants indigènes pour qui la revendication de l'ethnicité doit s'insérer dans un champ contestataire plus général devant provoquer un changement politique radical. Il s'agit pour eux, de rallier le MAS et son projet d'instauration d'État plurinational. Ce qu'ils parviennent à faire. En 2006, la CIDOB est réunifiée derrière une nouvelle direction soutenant le projet gouvernemental d'Evo Morales, élu quelques mois auparavant.

\section{Changements politiques et nouvelles perspectives de mobilisation collective}

\section{Un contexte national et international favorable à la question autochtone}

Jamais dans son histoire, en tant qu'organisation représentative, la CIDOB n'a soutenu si ouvertement un projet politique, autre que le sien, comme celui du gouvernement du président Morales. Il faut dire que ce dernier a multiplié les déclarations de bonnes intentions à l'égard des peuples autochtones et s'est autoproclamé défenseur de leurs droits sur la scène internationale. Cette position s'est traduite par plusieurs mesures. La Loi de Reconduction Communautaire de la Réforme Agraire (2006) devrait permettre d'accélérer les expropriations de terres illégalement acquises ou occupées et de les reverser en priorité aux communautés indigènes, originaires et paysannes. En 2007, le contrôle socio-environnemental des activités pétrolières et gazières réalisées sur les terres des peuples autochtones a été réglementé et ces derniers peuvent désormais revendiquer des droits sur l'exploitation minière exercée sur leurs terres ${ }^{11}$.

En septembre 2007, l'adoption de la Déclaration des droits des peuples autochtones par l'Organisation des Nations Unies provoque une liesse au sein des organisations ethniques du pays. La négociation de ce texte a duré plus de vingt ans et fût l'objet de multiples débats entre les peuples autochtones et les États. La Déclaration affirme notamment que les premiers ont le droit à l'autodétermination et qu'en vertu de celui-ci, ils ont le droit de déterminer librement leur statut politique et d'assurer tout aussi librement leur développement économique, social

11. Décrets suprêmes 29033, 29103 et 29117. 
et culturel (art. 3), d'être autonomes et de s'administrer eux-mêmes pour tout ce qui touche à leurs affaires intérieures et locales (art. 4). Les organisations ethniques de Bolivie présentent un projet de loi pour inclure cette déclaration dans la législation nationale et organisent plusieurs manifestations pour obtenir toutes les garanties de cette intégration.

Face à la mobilisation et l'engouement des peuples autochtones, le gouvernement de Morales élève les 46 articles de la Déclaration des Nations Unies sur les droits des peuples autochtones au rang de loi de la République (loi 3760 du 7 novembre 2007). Pour les organisations ethniques, cette mesure est un premier pas vers la formalisation du texte international en attendant sa traduction dans la nouvelle Constitution. Celle-ci tarde à se concrétiser. Les débats houleux entre le gouvernement et l'opposition engendrent des conflits sociaux importants. L'Assemblée Constituante est suspendue à plusieurs reprises et menacée de dissolution au cours des nombreux mois de son activité. Les organisations autochtones (notamment la CIDOB) et paysannes ont contribué au maintien et à la poursuite du processus en exerçant des pressions sur les délégué(e)s et en contribuant aux débats avec de nombreuses propositions. La constitution bolivienne est rédigée en octobre 2008 et approuvée par référendum populaire en janvier 2009.

\section{Un nouveau paradigme constitutionnel}

L'une des caractéristiques de la Constitution bolivienne est d'instaurer un « État unitaire de droit plurinational et communautaire » (art. 1). Elle garantit aux peuples autochtones la libre détermination, le droit à l'autonomie, à l'autogouvernement, à la culture, à la reconnaissance des institutions et à la consolidation des entités territoriales dans le respect de l'unité de l'État (art. 2). Elle reconnaît leurs systèmes de gouvernement (art. 11 et 26). Ces principes fondamentaux se déclinent en une cinquantaine d'articles généraux faisant référence à la question autochtone, soit un huitième des articles de la Constitution. Ces derniers traitent du modèle de l'État, des droits humains des peuples autochtones (politiques, accès à la santé et à la sécurité médicale, éducation), des juridictions et des entités territoriales ethniques, de l'autonomie indigène, des systèmes locaux de gouvernement autochtone, de la territorialité autochtone et de la gestion collective des ressources naturelles, de la protection des connaissances traditionnelles de la représentation et la participation politique des peuples autochtones au Tribunal Constitutionnel Plurinational, au Conseil Electoral Plurinational, aux conseils départementaux et municipaux ou encore du soutien de l'État à leurs projets économiques.

La Constitution conçoit une nouvelle catégorie sociale et politique, celle de « nations et peuples indigène originaire paysans » que constitue « toute collectivité humaine qui partage une identité culturelle, une langue, une tradition historique, des institutions, une territorialité et une cosmovision dont l'existence est antérieure à l'invasion coloniale espagnole » (art. 30). Il s'agit ici d'une innovation conceptuelle qui vient unifier, jusque dans l'usage du singulier ${ }^{12}$, des catégories ethniques régionales historiques dans la perspective de dépasser les différenciations terminologiques (indigène ou originaire ou paysan) et politiques

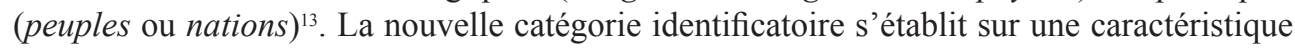

12. On peut remarquer l'accord du nombre n'instaurant qu'un « $\mathrm{s}$ » final à l'expression « indigène originaire paysans » pour renforcer l'idée d'une catégorie commune et unique.

13. Albó et Romero 2009. 
commune, celle de la préexistence précoloniale (art. 270 de la Constitution et article 5 de la Loi Cadre des Autonomies de 2010). Sa diversité interne légitime l'existence même de l'État plurinational. Cette innovation conceptuelle, qui a suscité d'intenses débats lors de l'Assemblée Constituante, est propre à la Bolivie et cette reformulation constitutionnalisée n'a d'autre sens que celui de «peuples autochtones » usité au niveau international.

L'adoption constitutionnelle d'un État plurinational conduit vraisemblablement la Bolivie vers un changement de gouvernance de l'autochtonie et de l'ethnicité. On observe que les principes dudit multiculturalisme libéral prédominant dans les années 1990 sont délaissés (Lacroix 2011). La Bolivie semble ainsi s'orienter vers un modèle politique inédit de type « post multiculturel » (Grey Postero 2007) qui considère pleinement la Déclaration des Nations Unies sur les Droits des Peuples Autochtones et appréhende la question ethnique comme une question politique centrale et transversale d'un nouveau projet sociétal.

\section{L'autonomie indigène, un premier pas dans la construction de l'État plurinational}

Pour instaurer l'État plurinational en Bolivie, le gouvernement d'Evo Morales estime qu'il ne faut pas moins d'une centaine de lois fondamentales. Le pays s'est donc engagé dans un processus de changement à long terme. Les peuples autochtones ont bien conscience des échéances et des défis. Ils restent vigilants quant à la mise en place du nouvel État et à l'application des droits constitutionnels qui les concernent.

Pour les peuples autochtones, en particulier ceux qui sont affiliés à la CIDOB, la mise en place des autonomies indigènes constitue un premier pas vers la construction effective de l'État plurinational. La Constitution bolivienne reconnaît les territoires ancestraux des peuples autochtones et les considère comme des entités territoriales inscrites dans la nouvelle organisation politico-administrative du pays. Au même titre que les autres types d'entités territoriales, elles constituent des circonscriptions juridictionnelles à part entière, assument des compétences (similaires à celles des municipalités) et jouissent d'un régime spécifique $\mathrm{d}$ 'autonomie indigène originaire paysanne qui peut s'appliquer au niveau municipal, régional et des TCO.

Ce régime et ses modalités d'attribution sont définis par la Loi Cadre des Autonomies et de Décentralisation (2010). Les entités territoriales autochtones doivent élaborer un « statut autonomique » qui devra être approuvé par référendum et par les deux tiers des votes de la population locale. Ces statuts doivent considérer les minorités non-autochtones en leur garantissant les droits établis par la Constitution. Des gouvernements autochtones pourront alors se constituer et exercer leurs pouvoirs de législation, délibération, réglementation et assumer les compétences qui leur sont attribuées (Lacroix 2010). À ce jour, onze municipalités et quelques TCO ont engagé une procédure pour adopter un régime d'autonomie indigène. Ainsi établie, l'autonomie indigène répond à la demande territoriale de la CIDOB.

À l'instar des autonomies indigènes, ce sont toutes les déclinaisons de l'État plurinational qui doivent être définies en Bolivie. La CIDOB compte bien s'assurer de l'application effective de la Constitution. Dans cette perspective et après avoir directement contribué à la situation politique actuelle générant de grands espoirs pour les peuples autochtones, la confédération indigène devrait encore occuper une place importante dans la vie politique bolivienne en organisant de nouvelles mobilisations et en continuant d'élaborer des propositions politiques. Elle devra probablement trouver de nouvelles formes d'utilisation politique de l'ethnicité 
pour s'assurer de la mise en place de l'État plurinational. Car si la Bolivie a été pionnière dans la reconnaissance des droits autochtones, ces derniers ne sont toujours pas en vigueur ${ }^{14}$.

\section{Références citées}

Acosta, Alberto et Esperanza Martinez (comp.), 2009. Plurinacionalidad. Democracia en la diversidad. Quito : Abya-Yala.

Albó, Xavier, 1991. « El retorno del Indio », Revista Andina 2 : 300-337.

Albó, Xavier et Carlos Romero, 2009. Autonomías Indígenas en la realidad boliviana y su nueva Constitución. La Paz : Vicepresidencia del Estado Plurinacional de Bolivia/PADEP-GTZ.

BARTh Fredrik, 1995 [1969]. « Les groupes ethniques et leurs frontières », in Philippe Poutignat, Jocelyne StreiffFenart, Théories de l'ethnicité, 203- 249. Paris : Presses Universitaires de France.

Bellier, Irène, 2007. « Le développement et les peuples autochtones : conflits de savoirs et enjeux de nouvelles pratiques politiques, in V. Geronimi, I. Bellier, J.-J. Gabas, M. Vernières et Y. Viltard, Savoirs et politiques de développement. Questions en débat à l'aube du XXI' siècle, 125-145. Paris : Karthala.

_-_-, 2009. « Autochtone », EspacesTemps.net, Mensuelles, http://espacestemps.net/document7583.html

Boccara, Guillaume, 2010. « Cet obscur objet du désir... multiculturel », Nuevo Mundo Mundos Nuevos (I) « Pouvoir et Différence », http://nuevomundo.revues.org/59975; (II) « indianité, citoyenneté et nation à l'ère de la globalisation néolibérale ", http://nuevomundo.revues.org/59991 ; (III) " ethnogenèse, ethnicisation et ethnification », http://nuevomundo.revues.org/59984.

Calla Ortega, Ricardo et Ramiro Molina Barrientos, 2000. « Los pueblos indígenas y la construcción de una sociedad plural », in Ricardo Calla Ortega, Ramiro Molina Barrientos et Cecilia Salazar de la Torre, Movimientos indigenas y pactos de género. Cuaderno de Futuro (5) : 9-38. La Paz : PNUD, IDH.

Castro Mantilla, María Dolores, 1997. La viva voz de las tribus. El trabajo del ILV en Bolivia. 1954-1980. La Paz : MDSP-VAIPO.

Centro de Estudios Jurídicos e Investigación Social (CEJIS), 1992. Territorios indígenas en el Oriente boliviano. Santa Cruz de la Sierra : CEJIS.

Chirif, Alberto, Pedro Garcia et Richard Chase Smith, 1991. El indigena y su territorio. Lima : OXFAM, COICA.

Confederación Indígena del Oriente, Chaco y Amazonia de Bolivia (CIDOB), 1996. « La Marcha Indígena y el papel de CIDOB. Un balance necesario ", Artículo Primero 2 : 88-92.

--_, 2000. Atlas. Territorios Indigenas en Bolivia. Situación de las Tierras Comunatarias de Origen (TCOs) y Proceso de Titulación. Santa Cruz de la Sierra : CIDOB-CPTI.

--_, 2008. Borrador de estatuto de autonomías indigenas. Documento de análisis y discusión. Santa Cruz de la Sierra : CIDOB.

De Certeau, Michel et Yves Materne, 1977. Le Réveil indien en Amérique latine. Paris : Cerf.

De VRIES, Aldert, 1998. Territorios Indígenas en las Tierras Bajas de Bolivia. Un análisis de su estado a 1998. Santa Cruz de la Sierra : CIDOB- CPTI, SNV.

Gagné, Natacha et Marie SAlaün, 2009. « De la difficulté à traiter les faits sociaux comme des 'choses' : l'Anthropologie et la question autochtone », Monde Commun 1, 2, http://www.mondecommun.com/ uploads/PDF/Gagnesalaun.pdf

14. Constat partagé par les représentants de l'Organisation des Nations Unies, de la Coordination Andine des Organisations Indigènes (CAOI) et de la Coordination des Organisations Indigènes du Bassin Amazonien (COICA) lors du Séminaire international sur « les Droits des Peuples Indigènes et leur viabilité dans un monde globalisé », organisé par l'Educación Radiofónica de Bolivia (Erbol) et la Asociación Latinoamericana de Educación Radiofónica (Aler), La Paz, 1 1 ${ }^{\mathrm{er}}$ au 4 juin 2010. 
Gallisot René, Mondher Kilani et Annamaria Rivera, 2000. L’imbroglio ethnique. Lausanne : Payot.

García Serrano, Fernando, 2001. «Política, Estado y diversidad cultural. La cuestión indígena en la región andina », Nueva Sociedad $173: 94-103$.

Gosselin, Gabriel, 2001. «Pour une ethnicité citoyenne », Cahiers internationaux de sociologie 1/2001, 110 : 121-130.

Grey Postero, Nancy, 2007. Now we are citizens : indigenous in postmulticultural Bolivia. Stanford : Stanford University Press.

Gros, Christian, 1999. «Ser diferente por (para) ser moderno, o las paradojas de la identidad. Algunas reflexiones sobre la construccón de una nueva frontera étnica en América latina », Análisis Político 36 : 3-20.

, 2007. «Ethniciser la politique, politiser l'ethnicité ? : le dilemme latino-américain », in G. Couffignal (éd.), Amérique latine 2007, 91-104. Paris : La Documentation Française.

Kymlicka, Will, 2001. La citoyenneté multiculturelle, une théorie libérale du droit des minorités. Paris : La Découverte, Collection Texte à l'appui/Politique et Société.

LABrousse, Alain, 1984. Le Réveil indien en Amérique andine. Lausanne : CETIM.

LACroIx, Laurent, 2005. Indigènes et Politique en Bolivie. Les stratégies chiquitanas dans le nouveau contexte de décentralisation participative, thèse de doctorat, Paris : IHEAL, Université Paris 3.

2006. «Bolivie : refondation du modèle politique national et tensions politiques », in Observatoire des changements en Amérique latine, Amérique latine 2006 : 83-98. Paris : La documentation Française.

2007. « Néoindigénisme d'État et ethnicisation en Bolivie (1994-2005) », in Denis Rolland et Joëlle Chassin (éds), Pour comprendre la Bolivie d'Evo Morales, 233-262. Paris : L'Harmattan.

2010. «Territorialité ethnique et agenda politique en Bolivie (1970-2010) », in V. Bretón et A. Roca (éds), Réformes agraires et gestion des ressources naturelles en Afrique et en Amérique latine, Actes de colloque, CD-Rom. Lleida : Universitat de Lleida.

--_-, 2011. «État plurinational et redéfinition du multiculturalisme en Bolivie », in Christian Gros et David Dumoulin, Le multiculturalisme au concret en Amérique latine. Paris : IHEAL, Presses de la sorbonne Nouvelle (à paraître).

LACroix, Laurent et Claude Le Gouill, 2010. « Hacia una nueva gobernanza territorial indígena en Bolivia », in Christian Gros et Jean Foyer (eds), Gobernanza económica y politica indígena en América latina, 41-74, Lima : IFEA.

Lavaud, Jean-Pierre, 1982. Identité et Politique : le courant Tupac Katari en Bolivie, CREDAL - ERSIPAL, document de travail 24. Paris : IHEAL.

--_, 2006. « Bolivie: vers l'anarchie segmentaire ? L'ethnicisation de la vie politique », Hérodote 123: 62-81.

Le Bot, Yvon, 1994. Violence de la modernité en Amérique latine. Indianité, société et pouvoir. Paris : Karthala, CNRS.

2009. La grande révolte indienne. Paris : Robert Laffont.

Lehm Aradaya, Zulema, 1999. Milenarismo y movimientos sociales en la Amazonia Boliviana. La busqueda de la Loma Santa y la Marcha Indígena por el Territorio y la Dignidad. Santa Cruz de la Sierra : APCOBCIDDEBENI-OXFAM AMERICA.

Lema, Ana María, 1997. Pueblos Indigenas de la Amazonía Boliviana. La Paz : PNUD, CAF, AIP, FIDA.

Libermann Cruz, Kitula et Armando Godínez Gutiérrez (coord.), 1992. Territorio y Dignidad. Pueblos indígenas y medio ambiente en Bolivia. La Paz : ILDIS, Nueva Sociedad.

Marinissen, Judith, 1995. Legislación boliviana y Pueblos Indigenas. Inventario y análisis en la perspectiva de las demandas indigenas. Santa Cruz de la Sierra : CEJIS, SNV. 
Morin, Françoise et Bernard Saladin D'Anglure, 1995. «L'ethnicité, un outil politique pour les autochtones de l'Arctique et de l'Amazonie », Etudes/Inuit/Studies 19 (1) : 37-68.

PACHeco, Diego, 1992. El indianismo y los indios contemporeanos en Bolivia. La Paz : HISBOL-MUSEF.

SAugestad, Sidsel, 2001. The Inconvenient Indigenous. Remote Area Development in Botswana, Donor Assistance, and the First People of the Kalahari. Uppsala : The Nordic Africa Institute.

Secretaría Nacional de Participación Popular (SNPP), 1997. El pulso de la democracia. Participación ciudadana y descentralización en Bolivia. Caracas : Nueva Sociedad.

TAYlor, Charles, 1994. Multiculturalisme. Différence et démocratie. Paris : Aubier.

Tully, James, 1999. Une étrange multiplicité : le constitutionnalisme à l'ère de la diversite. Sainte-Foy : Les Presses de l’Université Laval. 